\title{
5. HeilberufeSCIENCE-Symposium auf dem „Interprofessionellen Gesundheitskongress“ 2015 in Dresden
}

\author{
Jörg Klewer
}

Online publiziert: 19. Oktober 2014

(C) Springer-Verlag Wien 2014

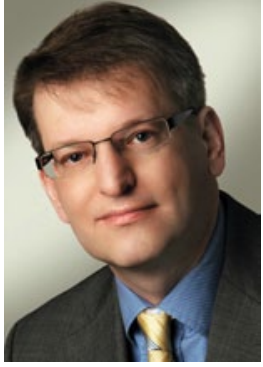

Jörg Klewer

Nachdem der „Interprofessionelle Pflegekongress“ im Jahr 2013 nach Dresden ,zurückgekehrt“ ist, wird er mit einem erweiterten inhaltlichen Angebot als „Interprofessioneller Gesundheitskongress" am 17. und 18. April 2015 wieder in Dresden stattfinden. Im Rahmen des Kongresses wird auch das 5. HeilberufeSCIENCE-Symposium ein Programmpunkt sein - wenn die Vorläufersymposien miteingerechnet werden, handelt es sich inzwischen genau genommen um die 8. Veranstaltung. Auch 2015 soll das HeilberufeSCIENCESymposium wieder aus einer Kombination von ,poster sessions" und Vorträgen bestehen.

Springer Medizin fördert mittlerweile seit Jahren durch die Verknüpfung der Heilberufe-Kongresse mit HeilberufeSCIENCE intensiv den wissenschaftlichen Austausch. Ich lade Sie daher herzlich ein, sich am 5. HeilberufeSCIENCESymposium mit einem Poster oder einem Vortrag zu beteiligen. Bitte reichen Sie Ihr Abstract bis zum 15.02.2015 ein. Weitere Informationen finden Sie im ,call for abstracts“ auf http://www.heilberufe-kongresse.de.

J. Klewer $(\triangle)$

Zwickau, Deutschland

E-Mail: joklewer@t-online.de
In dieser Ausgabe von HeilberufeSCIENCE bieten wir Ihnen 5 Beiträge zu unterschiedlichen Themen. Krau $\beta$ et al. untersuchen die Potenziale der stationär pflegerischen Betreuung von Demenzkranken und der Weiterentwicklung von Qualitätsmaßstäben bei der Betreuung dieser Patientengruppe. Meyer et al. stellen die methodischen Ansätze zur Untersuchung des Ernährungsmanagements in stationären Einrichtungen vor. Krell und Simon gehen auf die kritischen Einstellungen von Altenpflegeschülerinnen gegenüber dem Gebrauch von Computern und die Auswirkungen auf deren Computerwissen ein. Richter berichtet die Ergebnisse einer Onlinebefragung von Personalleitern deutscher Kliniken zum externen Rekrutierungsprozess von Oberärzten, und Fischer et al. präsentieren ihre Analyse der Internetauftritte von Hospizen in Ostdeutschland.

Ich lade Sie wieder ein, diese 5 wissenschaftlichen Arbeiten dieser Ausgabe von HeilberufeSCIENCE kritisch zu lesen und in den wissenschaftlichen Dialog einzutreten - und freue mich über Ihr Abstract für das 5. HeilberufeSCIENCE-Symposium in Dresden.

Zum Abschluss wünsche ich Ihnen eine schöne Weihnachtszeit und alles Gute für das Jahr 2015!

Ihr

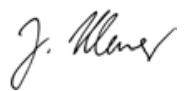

